Canad. Math. Bull. Vol. 23 (4), 1980

\title{
A NOTE ON FIXED POINT SETS AND WEDGES
}

\author{
BY \\ JOHN R. MARTIN ${ }^{(1)}$ AND SAM B. NADLER, JR.
}

\begin{abstract}
A space $Z$ is said to have the complete invariance property (CIP) provided that every nonempty closed subset of $Z$ is the fixed point set of some continuous self-mapping of $Z$. In this paper it is shown that there exists a one-dimensional contractible planar continuum having CIP whose wedge with itself at a specified point is contractible, planar, and does not have CIP.
\end{abstract}

1. Introduction. The problem of determining when a nonempty closed subset of a topological space $Z$ can be the fixed point set of a continuous self-mapping or homeomorphism of $Z$ has been investigated in [1] through [9]. In [9, p. 553] Ward defines a space $Z$ to have the complete invariance property (CIP) provided that each nonempty closed subset of $Z$ is the fixed point set of a continuous self-mapping of $Z$. Examples in [2, section 3] show that the wedge of two continua, each having CIP, does not necessarily have CIP, even when the continua are locally connected or one-dimensional and unicoherent. However, none of these continua is acyclic. In [2, 3.5] it is asked if CIP is preserved by wedging acyclic continua, one-dimensional acyclic continua, or contractible continua.

The purpose of this note is to show that there is a one-dimensional contractible planar continuum $X$ having CIP whose wedge with itself at a specified point does not have CIP. Thus, the questions in [2, 3.5] mentioned above are answered. In addition, the wedge is contractible and planar.

2. The example. Let $X=\left[\bigcup_{n=0}^{\infty} A_{n}\right] \cup S$ be the continuum in the plane $R^{2}$ pictured in Fig. 1, where $a_{0}=(-1,0), a_{n}=\left(-1,2^{-n}\right)$ for each $n=1,2, \ldots, A_{n}$ is the convex arc in $R^{2}$ from $(-3,0)$ to $a_{n}$ for each $n=0,1,2, \ldots$, and $S$ is the convex arc in $R^{2}$ from $a_{0}$ to $(0,0)$. Note that for each $n=1,2, \ldots$,

$$
A_{n}=\left\{(x, y) \in R^{2}:-3 \leq x \leq-1 \text { and } y=2^{-n-1}(x+3)\right\} \text {. }
$$

AMS Subject Classification (1970): Primary: 54F20, 54H25 Secondary: 54B99.

Key Words and Phrases: acyclic, complete invariance property, continuum, contractible, fixed point set, locally connected, one-dimensional, wedge.

Received by the editors November 27, 1978, in revised form March 1, 1979.

(1) The first author was partially supported by National Research Council of Canada grant no. A8205. 


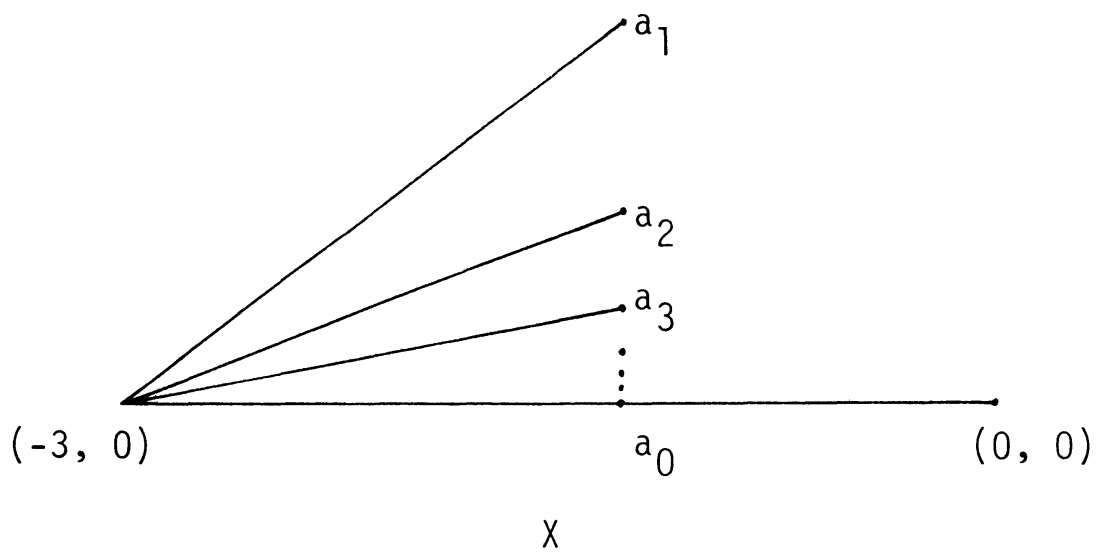

Figure 1

It is easy to see that $X$ is a one-dimensional contractible continuum. We leave it to the reader to verify that $X$ has CIP.

The wedge of $X$ with itself at $(0,0)$ is the quotient space obtained from the union of two disjoint copies of $X$ by identifying the points corresponding to $(0,0)$. This wedge is homeomorphic to the continuum $W$ drawn in Fig. 2,

$$
W=X \cup\left\{(-x, y) \in R^{2}:(x, y) \in X\right\} .
$$

It is easy to see that $W$ is contractible. We now show that $W$ does not have CIP. Let

$$
K=\{(x, y) \in W: x=-2 \text { or } x=+2\} .
$$

Suppose that there is a continuous function $f: W \rightarrow W$ such that $f$ has fixed point set equal to $K$. Then, using the uniform continuity of $f$, it follows that there exists $\varepsilon, 0<\varepsilon<1$, such that for

$$
q_{n}=\left(-2+\varepsilon, 2^{-n-1}[1+\varepsilon]\right) \in A_{n}, n=1,2, \ldots,
$$

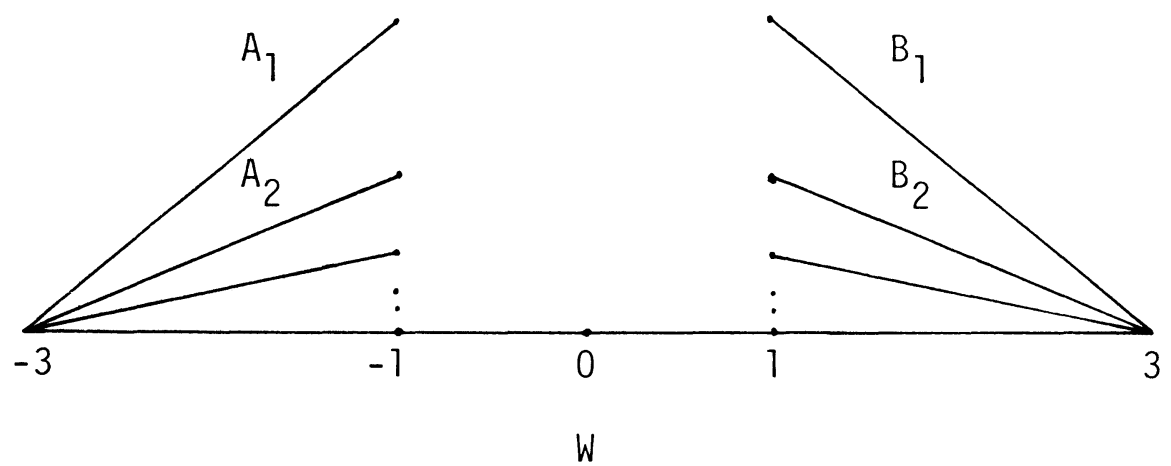

Figure 2 
$f\left(q_{n}\right) \in A_{n}$ for each $n=1,2, \ldots$ Hence, since $K$ is the fixed point set of $f$, the first coordinate of $f\left(q_{n}\right)$ is strictly less than $-2+\varepsilon$ for each $n=1,2, \ldots$ (otherwise, for some $n=1,2, \ldots, f$ would have a fixed point $(x, y) \in A_{n}$ such that $x \geq-2+\varepsilon)$. Thus, since $\left\{q_{n}\right\}_{n=1}^{\infty}$ converges to $q=(-2+\varepsilon, 0)$, we see that $f(q)$ is a point of the form $\left(x_{0}, 0\right)$ where $x_{0}<-2+\varepsilon$. Similarly, there exists $\varepsilon^{\prime}, 0<\varepsilon^{\prime}<1$, such that for $q^{\prime}=\left(2-\varepsilon^{\prime}, 0\right)$ we have $f\left(q^{\prime}\right)=\left(x_{0}^{\prime}, 0\right)$ where $x_{0}^{\prime}>$ $2-\varepsilon^{\prime}$. Let $\alpha$ be the arc in $W$ with end points $q$ and $q^{\prime}$. By comparing points of $\alpha$ with their images under $f$ while going along $\alpha$ from $q$ to $q^{\prime}$, it follows easily that $f$ must have a fixed point in $\alpha$. However, since $\alpha \cap K=\varnothing$, this is a contradiction.

REMARKs. In relation to the choice of $K$ at the beginning of the proof above, we mention that $\{(x, y) \in W: x=-1$ or $x=+1\}$ is the fixed point set of a continuous self-mapping of $W$.

Our continuum $X$ is one-dimensional and acyclic, but $X$ is not locally connected. Every one-dimensional, acyclic, locally connected continuum has CIP by [5, 3.1]; hence, any wedge of two such continua must also have CIP. However, we do not know if CIP is always preserved by wedging two locally connected continua which are contractible or planar. The reader is referred to [1] and [2] for other questions about locally connected continua and CIP.

\section{REFERENCES}

1. J. R. Martin, Fixed point sets of Peano continua, Pac. J. Math., 74 (1978), 163-166.

2. - and Sam B. Nadler, Jr., Examples and questions in the theory of fixed point point sets, Can. J. Math. 31 (1979), 1017-1032.

3. H. Robbins, Some complements to Brouwer's fixed point theorem, Israel J. Math., 5 (1967), 225-226.

4. H. Schirmer, On fixed point sets of homeomorphisms of the n-ball, Israel J. Math., 7, (1969), 46-50.

5. - Properties of fixed point sets on dendrites, Pac. J. Math., 36 (1971), 795-810.

6. - Fixed point sets of homeomorphisms of compact surfaces, Israel J. Math., 10 (1971), 373-378.

7. - Fixed point sets of homeomorphisms on dendrites, Fund. Math., 75 (1972), 117-122.

8. - Fixed point sets of polyhedra, Pac. J. Math., 52 (1974), 221-226.

9. L. E. Ward, Jr., Fixed point sets, Pac. J. Math., 47 (1973), 553-565.

Department of MATHEMATics

UNIVERSITY OF SASKATCHEWAN

SASKATOON, SASKaTCHEWAN

CANADA S7N OWO

DEPARTMENT OF MATHEMATICS

UNIVERSITY OF KENTUCKY

LEXINGTON, KENTUCKY 40506 